\title{
Bilateral Acute Anterior Uveitis after Intense Pulsed Light Treatment
}

Nark-Kyoung Rho'

Ho-Seok Sa ${ }^{2}$

${ }^{1}$ Leaders Aesthetic Laser and Cosmetic Surgery Center, Seoul, Korea

${ }^{2}$ Department of Ophthalmology, Asan Medical Center, University of Ulsan, College of Medicine, Seoul, Korea

\section{Correspondence}

Nark-Kyoung Rho

Leaders Aesthetic Laser and Cosmetic Surgery Center, THE CLASSIC500, 90, Neungdong-ro,

Gwangjin-gu, Seoul 00565, Korea

Tel.: +82-2-444-7585

Fax: +82-2-444-7535

E-mail: rhonarkahanmail.net

(C) Korean Society for Laser Medicine and Surgery

(c) This is an open access article distributed under the terms of the Creative Commons Attribution NonCommercial License (http://creativecommons.org/ licenses/by-nc/4.0) which permits unrestricted noncommercial use, distribution, and reproduction in any medium, provided the original work is properly cited.

\begin{abstract}
We report a 45-year old man who suffered acute ocular symptoms after an intense pulsed light treatment on the face and was diagnosed with bilateral acute uveitis. Given the increasing popularity of laser- and lightbased facial aesthetic procedures, treatment providers and ophthalmologists should be aware of the potential complication of anterior uveitis. Appropriate guidelines for standard practice of treatment around the eyes can help prevent potential laser- or lightassociated ocular complications.
\end{abstract}

\section{Key words}

Complications; Iritis; Lasers; Pulsed lights; Uveitis 


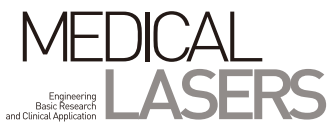

\section{INTRODUCTION}

Today a vast number of light and laser systems are used by physicians of different disciplines to address a variety of aesthetic challenges in the face. If improperly used, these modalities can inflict serious ocular injury on the patient, support personnel, and operator. ${ }^{1}$ It is important that medical service providers involved in operating these technologies be knowledgeable about the potential ocular complications of the unit being used. We present a case of bilateral acute anterior uveitis following aesthetic use of intense pulsed light (IPL) on the face.

\section{CASE REPORT}

A 45-year-old male presented with ocular redness, pain, photophobia, and decreased vision in the both eyes, which developed a day ago. The patient underwent an IPL procedure on the face including the upper eyelid by a general physician, and the ocular symptoms developed a few hours after the laser procedure. He had no remarkable medical history including uveitis, arthritis or oral and genital ulcerative lesions. On ophthalmic examination (Fig. 1) by a board-certified ophthalmologist, a corrected visual acuity was $20 / 20$ in both eyes, and intraocular pressure was $17 \mathrm{mmHg}$ in the right and $18 \mathrm{mmHg}$ in the left eye. A slit-lamp examination showed a ciliary injection of the conjunctiva and aqueous cells graded +2 in both eyes. Cornea remained clear and intact. The patient was diagnosed with the bilateral acute anterior uveitis, and treated with instillation of a topical corticosteroid four times a day. Ophthalmic symptoms and signs resolved one week after topical corticosteroid instillation.

\section{DISCUSSION}

The uveal tract comprises the iris and ciliary body anteriorly and the choroid posteriorly. Although a strict definition of uveitis, therefore, is limited to inflammation of these tissues, in common usage uveitis also includes inflammation of adjacent tissues, including the cornea, sclera, retina, and even the optic nerve. ${ }^{2}$ The types of uveitis are classified anatomically by the primary site of inflammation location leg, anterior, intermediate, posterior, and panuveitis. ${ }^{2}$ Onset (sudden vs. insidious), duration (limited vs. persistent), and course lacute, recurrent, or chronic) of uveitis are also important for diagnosis and categorization. Symptoms of uveitis vary based on the anatomic location, type of uveitis, duration of disease activity, extent of therapy, and presence or absence of prior sequelae. Common symptoms include blurred or distorted vision, pain, photophobia, floaters, photopsia, blind spots, and haloes. ${ }^{2}$ In the present case the patient's ocular symptoms and signs were confirmed by an ophthalmologist and the diagnosis of acute anterior uveitis was made. Uveitis can be associated with trauma, and systemic disease, such as sarcoidosis, autoimmune disease, infections (bacterial and parasitic), or in $25 \%$ of cases, has no associated cause. ${ }^{2}$ The present patient had no history suggesting infectious or autoimmune disorders related to uveitis, and the sequence of events leading to onset of ocular manifestations also supports the cause of acute uveitis is due to an IPL procedure. If further laboratory tests have been performed, a more definite exclusion of other etiologic factors could have been possible.

IPL devices use flashlamps and bandpass filters to emit polychromatic incoherent high-intensity pulsed light of determined wavelength spectrum, fluence, and
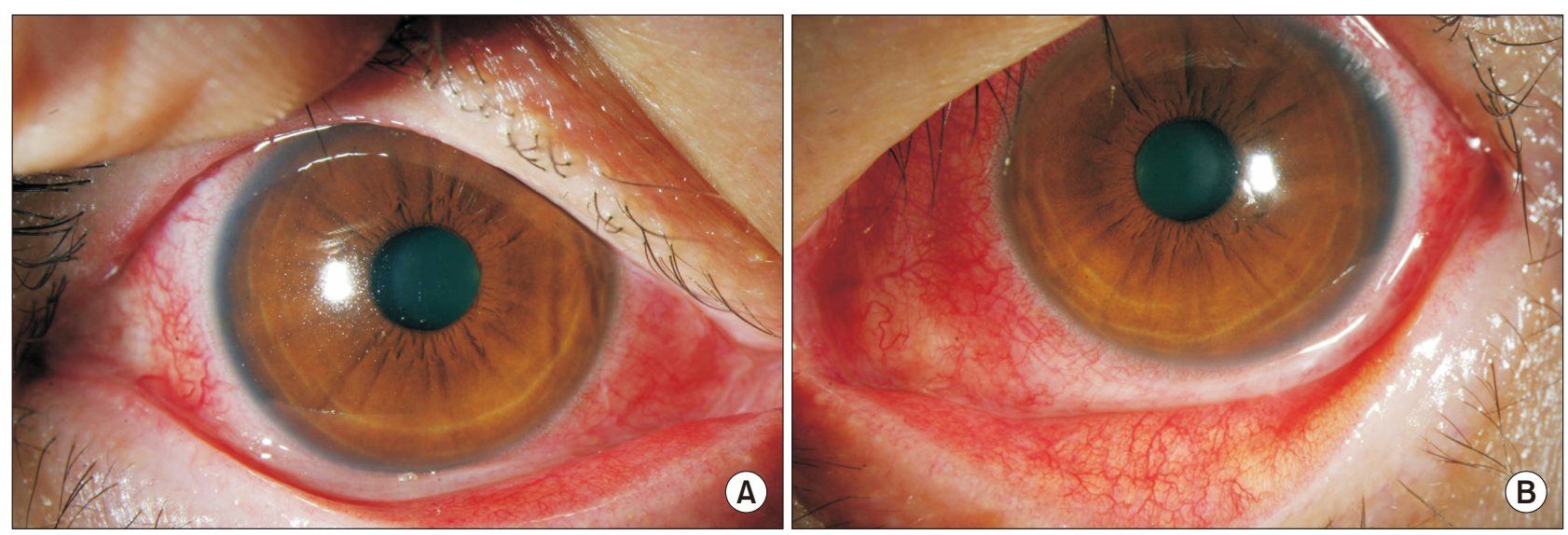

Fig. 1. Photographs of right (A) and left (B) pupils of the patient at the time of presentation. 
pulse duration. ${ }^{3}$ Although IPL is designed to specifically target pigmented skin tissue, other within the body has high pigment content and is therefore vulnerable during these procedures. ${ }^{4}$ The iris is one good example of noncutaneous tissue with high pigment content, ${ }^{5}$ and thus extremely susceptible to pigment-selective lasers or IPL if exposed. ${ }^{4}$ In the present case, the iris absorbed the broad-spectrum wavelengths, causing release of pigment and subsequent ocular inflammation. Cases of uveitis after aesthetic laser or light treatment have been reported in the literature. Pang et al. ${ }^{4}$ reported a case of bilateral ocular inflammation with permanent ocular damage after IPL photorejuvenation procedure. A similar case reported by Jewsbury and Morgan ${ }^{6}$ describes a 31 -year-old female who developed anterior uveitis and iris photoablation secondary to IPL procedure for facial aesthetics. Literature search found several cases $^{7-9}$ of acute anterior uveitis caused by laser -assisted hair removal of the eyebrows. Even though ocular adverse effects are not very frequent during aesthetic facial light-based procedures, they can lead to permanent ocular dysfunction and visual impairment. Upper eyelid skin is extremely thin and thus should not be directly irradiated by pigment-treating lasers or IPL. If upper eyelids have to be treated, patients should wear scleral protective lenses, possibly made with stainless steel, so that the energy of IPL can be dispersed. ${ }^{10}$

Given the fact that IPL procedure are frequently offered by physicians lack of proper laser safety education, nonmedically trained clinic staffs, dentists, doctors of oriental medicine, or even non-medical beauty care providers, the prevalence of adverse events including ocular complications are increasing. The authors would like to emphasize the importance of adequate training, sufficient clinical experience, and most of all, a basic understanding of ocular hazards in performing laser- or light-based facial aesthetic procedures.

\section{REFERENCES}

1. Sayed MS, Ko MJ, Ko AC, Lee WW. Ocular damage secondary to lights and lasers: how to avoid and treat if necessary. World J Ophthalmol 2014;4:1-6.

2. Dunn JP. Uveitis. Prim Care 2015;42:305-23.

3. Babilas P, Schreml S, Szeimies RM, Landthaler M. Intense pulsed light (IPL): a review. Lasers Surg Med 2010;42:93-104.

4. Pang AL, Wells K. Bilateral anterior uveitis after intense pulsed light therapy for pigmented eyelid lesions. Dermatol Surg 2008;34:1276-9.

5. Kels BD, Grzybowski A, Grant-Kels JM. Human ocular anatomy. Clin Dermatol 2015;33:140-6.

6. Jewsbury H, Morgan F. Uveitis and iris photoablation secondary to intense pulsed light therapy. Can J Ophthalmol 2012;47:e134.

7. Yalçındağ FN, Uzun A. Anterior uveitis associated with laser epilation of eyebrows. J Ophthalmic Inflamm Infect 2013;3:45.

8. Gunes A, Yasar C, Tok L, Tok O. Two cases of anterior uveitis after laser eyebrow epilation. Cornea 2015;34:101-2.

9. Karabela Y, Eliaçık M. Anterior uveitis following eyebrow epilation with alexandrite laser. Int Med Case Rep J 2015;8:1779.

10. Ricci LH, Navajas SV, Carneiro PR, Söderberg SA, Ferraz CA. Ocular adverse effects after facial cosmetic procedures: a review of case reports. J Cosmet Dermatol 2015;14:145-51. 